Enhancement by electric field of high-speed photoconductivity in AlGaN / GaN heterostructures

B. A. Danilchenko, S. E. Zelensky, E. A. Drok, A. E. Belyaev, V. A. Kochelap, H. Lüth, and S. A. Vitusevich

Citation: Appl. Phys. Lett. 90, 152102 (2007); doi: 10.1063/1.2721126

View online: https://doi.org/10.1063/1.2721126

View Table of Contents: http://aip.scitation.org/toc/apl/90/15

Published by the American Institute of Physics

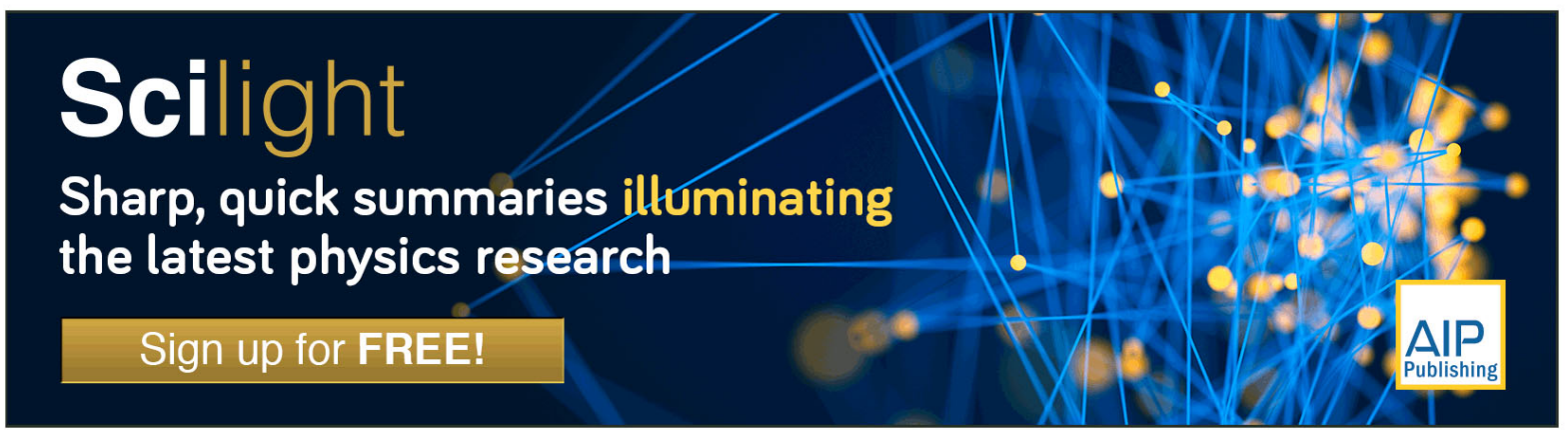




\title{
Enhancement by electric field of high-speed photoconductivity in AIGaN/GaN heterostructures
}

\author{
B. A. Danilchenko, S. E. Zelensky, and E. A. Drok \\ Institute of Physics, NASU, Pr. Nauki 41, 03028 Kiev, Ukraine
}

A. E. Belyaev and V. A. Kochelap

Institute of Semiconductor Physics, NASU, Pr. Nauki 45, 03028 Kiev, Ukraine

\begin{abstract}
H. Lüth and S. A. Vitusevich ${ }^{\text {a) }}$
Institute of Thin Films and Interfaces and CNI-Center of Nanoelectronic Systems for Information Technology, Research Centre Jülich, 52425 Jülich, Germany
\end{abstract}

(Received 6 October 2006; accepted 7 March 2007; published online 9 April 2007)

\begin{abstract}
The authors report a large response in the conductivity of $\mathrm{AlGaN} / \mathrm{GaN}$ heterostructures to a $10 \mathrm{~ns}$ UV laser pulses. The dynamics of the conductivity response follows the time evolution of the laser pulse. This fast photoconductivity component shows a remarkable enhancement in high electric fields. For the field $\approx 15 \mathrm{kV} / \mathrm{cm}$, it increases by at least one order of magnitude at temperatures of 4.2 and $300 \mathrm{~K}$. This photoconductivity enhancement is found to be related to the hot electron effect. The authors propose an explanation for the observed phenomena. The mechanism of the photoconductivity enhancement involves nonequilibrium LO phonons generated by hot carriers.

(C) 2007 American Institute of Physics. [DOI: 10.1063/1.2721126]
\end{abstract}

The basic elements of optoelectronic systems include an emitter and a detector operating in the same spectral range. For short-wavelength light (blue and UV), effective emitters (light-emitting diodes and laser diodes) have already been developed on the basis of group-III nitride materials. ${ }^{1-3}$ The second component of blue and UV optoelectronics, the photodetectors, needs to be considerably improved. Large and fast photoconductivity response effects are preferable for exploitation in UV detectors. In this letter, we report the effect of nanosecond response and enhanced photoconductivity revealed in the $\mathrm{AlGaN} / \mathrm{GaN}$ heterostructures in high electric fields.

The investigated devices were fabricated from $\mathrm{Al}_{0.33} \mathrm{Ga}_{0.67} \mathrm{~N} / \mathrm{GaN}$ undoped heterostructures grown by metal-organic chemical-vapor deposition on $\mathrm{SiC}$ and sapphire substrates. The conducting channel with twodimensional electron gas was formed at the $\mathrm{AlGaN} / \mathrm{GaN}$ interface due to spontaneous and piezoelectric polarization effects. Lateral devices with the same width $(100 \mu \mathrm{m})$ and different intercontact distances (from 10 to $25 \mu \mathrm{m}$ ) were studied. Standard ohmic contacts were processed by $\mathrm{Ti} / \mathrm{Al} / \mathrm{Ti} / \mathrm{Au}$ metallization and annealing for $40 \mathrm{~s}$ at $800{ }^{\circ} \mathrm{C}$. Photoconductivity was investigated in two regimes. Weak electric fields were applied in a steady-state regime. To reach high electric fields and avoid the thermal effects resulting in sample destruction, we used an electric pulse regime of $100 \mathrm{~ns}$ duration. In both regimes, the device was illuminated by an incident $10 \mathrm{~ns}$ laser beam from the top $\mathrm{Al}_{0.33} \mathrm{Ga}_{0.67} \mathrm{~N}$ layer. The energy quantum of the $\mathrm{N}_{2}$ laser was $3.7 \mathrm{eV}$, which exceeds the band gap of $\mathrm{GaN}$. The kinetics of photoconductivity was investigated with a time resolution of $0.1 \mathrm{~ns}$. Measurements were performed at 4.2 and $300 \mathrm{~K}$.

We found that heterostructures grown on sapphire, as well as on $\mathrm{SiC}$ substrate, demonstrate a similar time evolution of conductivity induced by the laser. The typical wave

\footnotetext{
${ }^{a)}$ Electronic mail: s.vitusevich@fz-juelich.de
}

forms of the current and photoconductivity measured in the pulse regimes are shown in Figs. 1(a) and 1(b), respectively. Two well-pronounced components in the conductivity alteration were observed in both pulse and steady-state electrical regimes. The first one is very sharp and practically follows the laser intensity distribution over time. For this fast component, there was no essential conductivity delay with respect to the laser pulse. The second component displays a very long conductivity tail with the characteristic time of $0.1-1 \mathrm{~s}$, which can be related to the well-known persistent conductivity. ${ }^{4}$

Before discussing the results, we define the increasing part of the conductivity due to interband light absorption

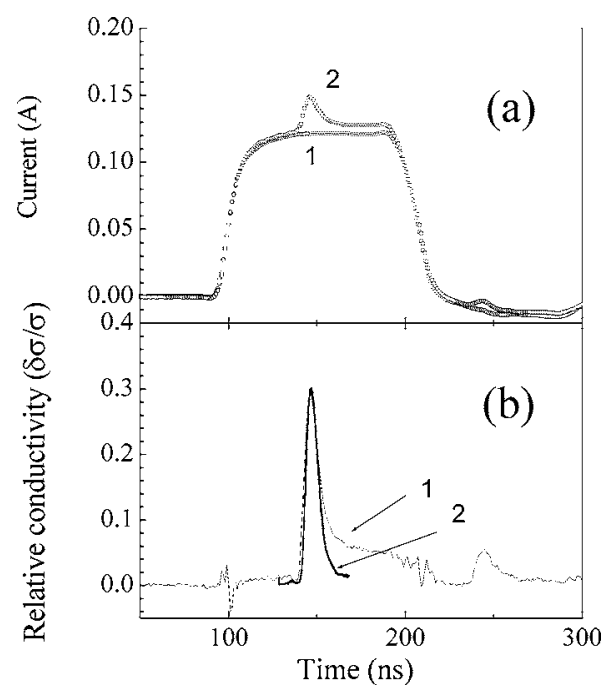

FIG. 1. (a) Wave forms of the current: (1) in the dark and (2) under the $10 \mathrm{~ns}$ UV laser illumination. (b) (1) Photoconductivity related to the dark conductivity as a function of time; pulse electric field is $E=4.7 \mathrm{kV} / \mathrm{cm}$. Results are presented for a heterostructure on $\mathrm{SiC}$ substrate at the ambient temperature $4.2 \mathrm{~K}$. (2) Wave form of the laser emission. Small secondary peaks in (a) and (b) are related to signals reflected in coaxial cables. 


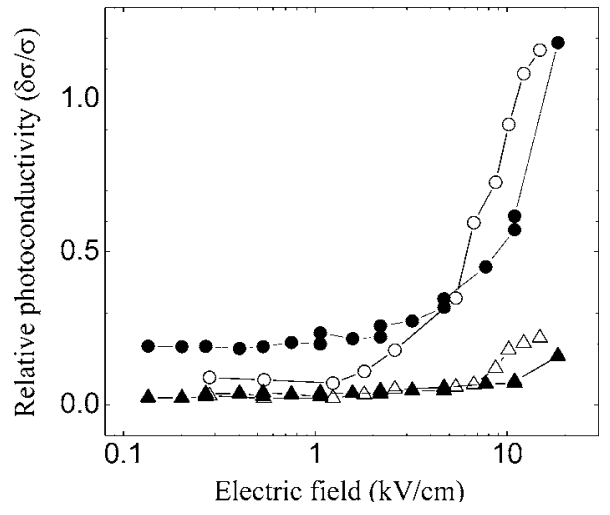

FIG. 2. Relative photoconductivity vs electric field for the fast (circles) and slow components (triangles) for $\mathrm{AlGaN} / \mathrm{GaN}$ on $\mathrm{SiC}$ substrate. Filled symbols, $300 \mathrm{~K}$; open symbols, $4.2 \mathrm{~K}$.

with the excitation of nonequilibrium carriers (electrons and holes) as

$$
\delta \sigma=e \mu_{e}(E) \delta n_{e}+e \mu_{h}(E) \delta n_{h} .
$$

Here $e$ is the elementary charge, and $\mu_{e, h}$ and $\delta n_{e, h}$ are the electron or hole mobility and concentrations, respectively (obviously, $\delta n_{e}=\delta n_{h}$ ). In general, $\mu_{e, h}$ is dependent on the electric field $E$ applied. The electron mobility in the conducting layers is estimated to be $\cong 10^{3} \mathrm{~cm}^{2} / \mathrm{V} \mathrm{s},{ }^{5}$ while in a GaN the hole mobility is at least ten times lower than the electron mobility. ${ }^{6}$ Therefore, the contribution of the second term of Eq. (1) can be neglected. If the laser pulse duration exceeds the recombination time of nonequilibrium carriers, the photoinduced increase in the electron concentration can be determined as

$$
\delta n_{e}=I \tau \kappa \alpha,
$$

where $I$ is the photon flux density (photon $/ \mathrm{cm}^{2} \mathrm{~s}$ ), $\alpha$ is the light absorption coefficient, $\tau$ is the lifetime of the photoexcited carriers, and $\kappa$ is the quantum efficiency.

Results of photoconductivity measurements in the pulse regime for the most interesting case of the fast component are presented in Fig. 2 by circles. For a comparison, we also show the slow component in the figure. The slow component was measured at $30 \mathrm{~ns}$ delay with respect to the laser pulse. The electric pulse regime allows us to measure the photoresponse at high fields of up to $13 \mathrm{kV} / \mathrm{cm}$ at $4.2 \mathrm{~K}$ and $18 \mathrm{kV} / \mathrm{cm}$ at $300 \mathrm{~K}$. In Fig. 2, the ratio of photoconductivity $\delta \sigma(E)$ to the dark conductivity $\sigma(E)$ is plotted against the electric field $E$. Such a presentation of the experimental data shows that the photoconductivity effect is large and the lifetime of photogenerated carriers is dependent on the field. Indeed, in accordance with Eqs. (1) and (2), we obtain

$$
\frac{\delta \sigma(E)}{\sigma(E)}=\frac{e \mu_{e}(E) I \kappa \alpha d \tau(E)}{e \mu_{e}(E) n_{e}} \propto \tau(E),
$$

where $n_{e}$ is the number of electrons in the dark and $d$ is the conducting channel thickness. As can be seen from Fig. 2 and relation (3), the lifetime $\tau$ remains constant in weak electric fields up to $1 \mathrm{kV} / \mathrm{cm}$. At higher fields, the recombination time increases rapidly without saturation up to a field of $18 \mathrm{kV} / \mathrm{cm}$. Figure 2 demonstrates that the behavior of the lifetime with the electric field is similar for 4.2 and $300 \mathrm{~K}$.

To clarify the physical origin of the behavior of the lifetime discussed above, we performed the following additional

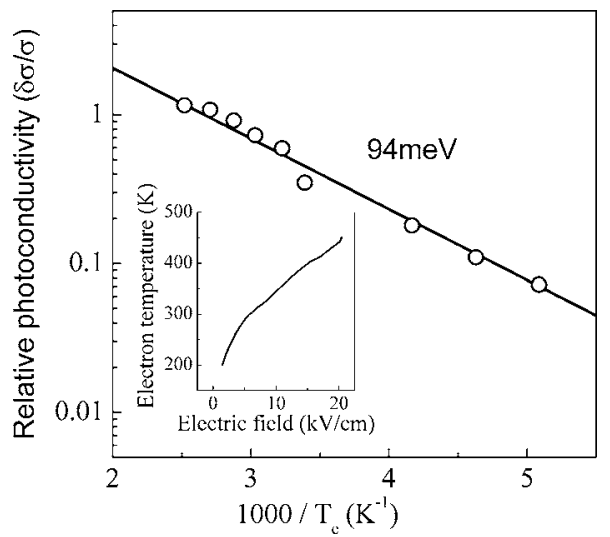

FIG. 3. Fast component of photoconductivity normalized to the dark conductivity at the same electric field and presented as a function of inverse electron temperature. Sample temperature is $4.2 \mathrm{~K}$. The solid line is the exponential law according to Eq. (4).

experiments. Using the mobility comparison method, ${ }^{7,8}$ we deduced the field dependence of the electron temperature in the conducting channel $T_{e}(E)$, which is shown in the inset to Fig. 3. Then, using the obtained curve $T_{e}=f(E)$, the results of the measurements shown in Fig. 2 can be presented in the coordinates $\ln (\delta \sigma / \sigma)$ vs $1 / T_{e}$ (see Fig. 3). From Fig. 3, it can be seen that the value of $\ln (\delta \sigma / \sigma)$ demonstrates an almost lineal dependence on $1 / T_{e}$ and follows an empirical Arrhenius law:

$$
\frac{\delta \sigma}{\sigma} \approx \exp \left(-\frac{\varepsilon}{k_{B} T_{e}}\right),
$$

where $\varepsilon$ is the activation energy and $k_{B}$ is the Boltzmann constant. Fitting of the experimental data by Eq. (4), as shown in Fig. 3 by the solid line, allows us to obtain the activation energy $\varepsilon=94 \mathrm{meV}$. The obtained value of the activation energy practically coincides with the energy of the LO phonons in $\mathrm{GaN}, 92 \mathrm{meV}$, which was deduced from hot carrier transport experiments. ${ }^{7,9}$ About the same phonon energy of $91 \mathrm{meV}$ was obtained from optical measurements. ${ }^{10}$

The coincidence of the activation energy extracted from photoconductivity measurements and the LO-phonon energy seems to be unexpected. There is no obvious direct relation between the optical phonons and the observed photoconductivity enhancement at high electric fields. Below we propose some arguments for a possible mechanism that provides a qualitative understanding of the phenomenon observed.

We start by recalling that with illumination of our structure, there are two contributors into the electric current: twodimensional (2D) electrons confined near the $\mathrm{AlGaN} / \mathrm{GaN}$ interface and photogenerated carriers distributed over an extended layer $\left(\approx \alpha^{-1}\right)$.

The 2D electrons primarily determine the dark conductivity of the structures. Electric power $P_{E}$ supplied to the 2D electrons is dissipated via acoustic and optical phonon emissions. With increasing electrical field, spontaneous emission of LO phonons dominates and balances the input power $P_{E}$. We can thus write the following relations:

$$
P_{E}=\hbar \omega_{0} \frac{n_{e}\left(\varepsilon>\hbar \omega_{0}\right)}{\tau_{\mathrm{em}}}=\frac{\hbar \omega_{0} N}{\tau_{\mathrm{dec}}} \propto \exp \left(-\frac{\hbar \omega_{0}}{k_{B} T_{e}}\right) .
$$

Here $\hbar \omega_{0}$ is the energy of LO phonons, $\tau_{\mathrm{em}}$ is the time of spontaneous emission of the phonons, and $n_{e}\left(\varepsilon>\hbar \omega_{0}\right)$ is the 
number of the electrons, which have enough energy to emit optical phonons. The latter is approximately proportional to $\exp \left(-\hbar \omega_{0} / k_{B} T_{e}\right)$. For the case under consideration, the input electric power is equal to the rate of dissipation of the total LO phonon energy $\hbar \omega_{0} N / \tau_{\mathrm{dec}}$, where $N$ and $\tau_{\mathrm{dec}}$ are the number of LO phonons and characteristic time of their decay, respectively. The effect of the reabsorption of optical phonons is negligibly small for $E<15 \mathrm{kV} / \mathrm{cm}$, according to our results reported in Ref. 7 due to the absence of phonon accumulation. Emitted optical phonons spontaneously decay into acoustic phonons. The estimated time of spontaneous decay of LO phonons is from 1 to $3-4$ ps. ${ }^{11,12}$ Taking into account the dispersion of the phonon in wurtzite $\mathrm{GaN},{ }^{\mathrm{P}}$ and the momentum and energy conservation laws, leads us to the conclusion $^{14}$ that LO phonons decay into TO and LA phonons: $\mathrm{LO} \rightarrow \mathrm{TO}+\mathrm{LA}$. Further decay of TO phonons results in secondary acoustic phonons with average energies of $30-35 \mathrm{meV}$. Thus, the hot electrons produce a flux of shortwavelength acoustic phonons propagating through the structures.

For photogenerated carriers, we may assume the Shockley-Reed ${ }^{15}$ recombination mechanism, which involves carrier capture on shallow traps. For unintentionally doped $\mathrm{GaN}$, the energy of such traps is in the range of $20-40 \mathrm{meV}$ below the conduction band. ${ }^{16}$ As a result, the energy of the secondary phonons produced by the hot electrons is comparable to the ionization energies of the shallow traps. Thus, the secondary phonons can be absorbed by electrons captured on the traps, resulting in trap depopulation and electron return to the conduction band. On the whole, this leads to an increase of the effective lifetime $\tau(E) .{ }^{17}$ The rate of trap depopulation is proportional to the flux of the secondary acoustic phonons $J_{\text {ac ph }}$, i.e., $\tau(E) \approx J_{\text {ac ph }}$. In quasistationary state, the acoustic phonon flux is determined by the rate of LOphonon decay: $N / \tau_{\mathrm{dec}}=J_{\mathrm{ac}}$ ph. Finally, we obtain $\tau(E)$ $\approx \exp \left(-\hbar \omega_{0} / \kappa_{B} T_{e}\right)$. This result qualitatively explains the experimental data presented in Fig. 3.

In conclusion, we observed a large photoresponse of $\mathrm{AlGaN} / \mathrm{GaN}$ heterostructures with nanosecond-scale time resolution. It was found that the photoconductivity increases by more than one order of magnitude at $15 \mathrm{kV} / \mathrm{cm}$. The results obtained demonstrate that nonequilibrium phonons emitted by hot electrons confined at the $\mathrm{AlGaN} / \mathrm{GaN}$ interface can play a key role in the observed phenomenon of photoresponse enhancement in high electric fields.

This work was supported by the Program of Targeted Initiatives established by STCU (USA) and NASU (Ukraine) under Grant No. 3922 and DFFD Grant No. F7/379 and by the Deutsche Forschungsgemeinschaft (Project No. KL 1342).

${ }^{1}$ J. W. Orton and C. T. Foxon, Rep. Prog. Phys. 61, 1 (1998).

${ }^{2}$ S. J. Pearton, J. C. Zolper, R. J. Shui, and R. J. Ren, J. Appl. Phys. 86, 1 (1999).

${ }^{3}$ S. Nakamura, S. Pearton, and G. Fasol, The Laser Diode (Springer, Berlin, 2000), p. 368.

${ }^{4}$ V. V. Ursaki, M. Tiginyanu, P. C. Ricci, A. Anedda, S. Habbard, and D. Pavlidis, J. Appl. Phys. 94, 3875 (2003).

${ }^{5}$ B. A. Danilchenko, S. E. Zelensky, E. Drok, S. A. Vitusevich, S. V. Danylyuk, N. Klein, H. Lüth, A. E. Belyaev, and V. A. Kochelap, Phys. Status Solidi B 243, 1529 (2006).

${ }^{6}$ Erik L. Waldron, John W. Graff, and E. Fred Schubert, Appl. Phys. Lett. 79, 2737 (2001).

${ }^{7}$ B. A. Danilchenko, S. E. Zelensky, E. Drok, S. A. Vitusevich, S. V. Danylyuk, N. Klein, H. Lüth, A. E. Belyaev, and V. A. Kochelap, Appl. Phys. Lett. 85, 5421 (2004).

${ }^{8}$ N. M. Stanton, P. Hawker, A. J. Kent, T. S. Cheng, and C. T. Foxon, Phys. Status Solidi A 176, 369 (1999).

${ }^{9}$ L. Ardaravicius, A. Matulionis, J. Liberis, O. Kiprijanovic, M. Ramonas, L. F. Eastman, J. R. Shealy, and A. Vertiatchikh, Appl. Phys. Lett. 83, 4038 (2003).

${ }^{10}$ V. W. L. Chin, T. L. Tansley, and T. Osotchan, J. Appl. Phys. 75, 7365 (1994).

${ }^{11}$ M. Ramonas, A. Matulionis, J. Liberis, L. Eastman, X. Chen, and Y.-J. Sun, Phys. Rev. B 71, 075324 (2005).

${ }^{12}$ Kejia Wang, John Simon, Niti Goel, and Debdeep Jena, Appl. Phys. Lett. 88, 022103 (2006).

${ }^{13}$ M. Sanati and S. K. Estreicher, J. Phys.: Condens. Matter 16, L327 (2004).

${ }^{14}$ B. K. Ridley, J. Phys.: Condens. Matter 8, L511 (1996).

${ }^{15}$ R. A. Smith, Semiconductors (Cambridge University Press, Cambridge, 1978), p. 523.

${ }^{16}$ Properties of Advanced Semiconductor Materials (GaN, AlN, InN, BN, $\mathrm{SiC}$, SiGe), edited by M. E. Levinshtein, S. L. Rumyantsev, and M. S. Shur (Wiley, New York, 2001), p. 194.

${ }^{17}$ Effect of depopulation of shallow traps by acoustic phonons has been directly observed in silicon: B. A. Danil'chenko and S. I. Komirenko, JETP Lett. 54, 380 (1991). 